\title{
Technique selection of ureteroileal anastomosis in hautmann ileal neobladder with chimney modification: Reliability of patient-based selection strategy and its impact on ureteroentric stricture rate
}

\author{
Dejan Djordjevic $^{1}$, Svetomir Dragicevic ${ }^{1}$, Marko Vukovic ${ }^{2}$ \\ ${ }^{1}$ Urology Clinic, Euromedik General Hospital, Belgrade, Serbia; \\ 2 Urology clinic, Clinical centre of Montenegro, Podgorica, Montenegro.
}

\begin{abstract}
Summary Objective: We aimed to establish the reliability of technique selection strategy for ureteroileal anastomosis (Bricker vs. Wallace) by comparing perioperative outcomes, complications, and anastomotic stricture rate in a contemporary series of patients who underwent open radical cystectomy followed by reconstruction of modified Hautmann neobladder.

Materials and methods: A total of 60 patients underwent radical cystectomy and modified Hautmann neobladder, of whom 30 patients (group I) with Bricker anastomotic technique were compared to 30 matched paired patients with end-to-end ureteroileal anastomosis (group II). Long-term results, including ureteroileal stricture (UIS) and postoperative complication rate at two year follow up were available. The choice of anastomosis type was successively based on chimney size, ureteral length after retro-sigmoidal tunneling and diameter of distal ureter. Postoperative complications were graded according to the Clavien-Dindo system.

Results: Ureteroileal stricture rate was $6.6 \%$ in group I vs. $0 \%$ in group II, after three months ( $p<0.05)$, while anastomotic leakage rate was $6.6 \%$ vs. $3.3 \%$ (group I vs group II) between the two groups for the same follow up period ( $p>0.05)$. High-grade complications (Clavien III-V) were more in Bricker group as compared to Wallace group and the difference was significant (20\% vs $10.3 \%, p=0.03$ ).

Conclusion: Our preliminary outcomes demonstrate that this selection strategy seems to be clinically reliable, with lower incidence of postoperative complications in Wallace group.
\end{abstract}

KEY WORDS: Orthotopic bladder substitution; Urinary diversion; Reconstructive urology; Selection strategy; Surgical technique.

Submitted 25 May 2021; Accepted 25 June 2021

\section{INTRODUCTION}

In both male and female patients, orthotopic bladder substitution has become the preferred method of urinary diversion post radical cystectomy (RC) for malignant disease (1). Among different reconstructive modalities, ileal neobladder with Hautmann or Studer reservoir is a frequent orthotopic diversion and several modified techniques have been described (2-6).

The standard technique for uretero-enteric anastomosis is the Bricker ureteral implantation in an end-to-side fashion using running sutures (7). According to the literature, the ureteroileal stricture (UIS) rate using this technique ranges between 3\%-20\% (7-10). A frequently used anastomotic technique in urinary diversions is that described by Wallace, in which the end of the intestine is sutured to the end of the ureter (11). It is already known that this technique has the lowest complication rate comparing to other ureterointestinal anastomosis (12), including its usage in orthotopic bladder reservoirs $(13,14)$.

Nevertheless, only a few studies favour this technique in orthotopic neobladder $(5,6,15)$.

The objective of this study was to establish the reliability of technique selection strategy for ureteroileal anastomosis, based upon patients characteristics; additionally, we aimed to compare perioperative outcomes and ureteroileal anastomotic stricture rate in a contemporary series of patients who underwent open RC followed by reconstruction of modified Hautmann neobladder.

\section{MATERIALS AND METHOdS}

\section{Study design and patients}

We compared 30 matched paired patients who underwent Hautmann neobladder with single chimney and Bricker anastomotic technique $(2,3)$ with 30 matched paired patients who underwent Hautmann neobladder with chimney modification consisting of a longer ureteral spatulation $(3-4 \mathrm{~cm})$ combined with end-to-end ureteroileal anastomosis (Wallace type I) and 6-8 $\mathrm{cm}$ long isoperistaltic tubularised chimney (16).

Long-term results, including uretero-ileal stenosis (UIS) and postoperative complications rate (graded according to Clavien-Dindo system) at 2-year follow-up, were available for analysis.

The main differences between techniques were the length of the ureteral spatulation, the chimney size and the endto-end running suture ureteroileal anastomosis (Figure 1). Patient characteristics included three aspects: ureteral length after retro-sigmoidal tunneling, chimney size and diameter of distal ureter after dissection and preparation for anastomosis. When the ureteral length was similar on 
Figure 1.

Differences between two techniques:

a) modified Hautmann neobladder with long chimney and Bricker ureteroileal anastomosis (group l);

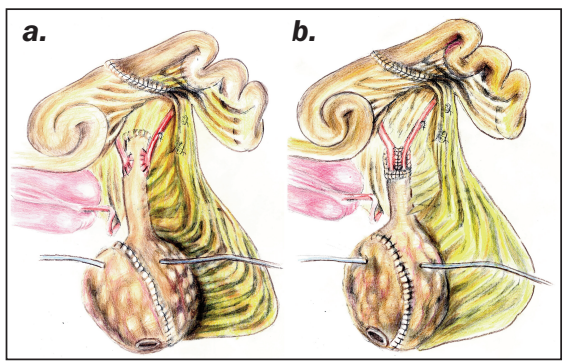

b) our modified technique with short afferent limb, similar ureteral length on both sides and Wallace anastomotic type (group II).

both sides, Wallace was preferred; when disparate, Bricker was performed $(12,17)$. Moreover, if the ureters were transected at the level of common iliac vessels [diffuse carcinoma in situ (CIS)], chimney length was 10-12 $\mathrm{cm}$ and Bricker anastomosis was performed $(2,3)$; if ureters were divided more distally, as close to the bladder as possible, Wallace anastomosis on 6-8 $\mathrm{cm}$ long chimney was preferred (16). The third decision was based on the diameter of distal ureteral end, after the ureter has been divided and prepared for anastomosis. When distal ureteral end was more than double size of normal diameter (long-standing hydronephrosis), Wallace was preferred. If distal end was of a normal caliber, the choice of anastomotic technique was based upon other two variables. The inclusion and exclusion criteria are presented in the patient flowchart (Figure 2).

Bladder cancer $(\mathrm{BCa})$ patients scheduled for definitive treatment were recruited from the Urology Clinic at the Clinical Centre of Serbia. The surgical protocol had been approved by the University of Belgrade Institutional review board and registered with the Ethical committee of

Figure 2.

Patient flowchart describes selection criteria for surgical approach.

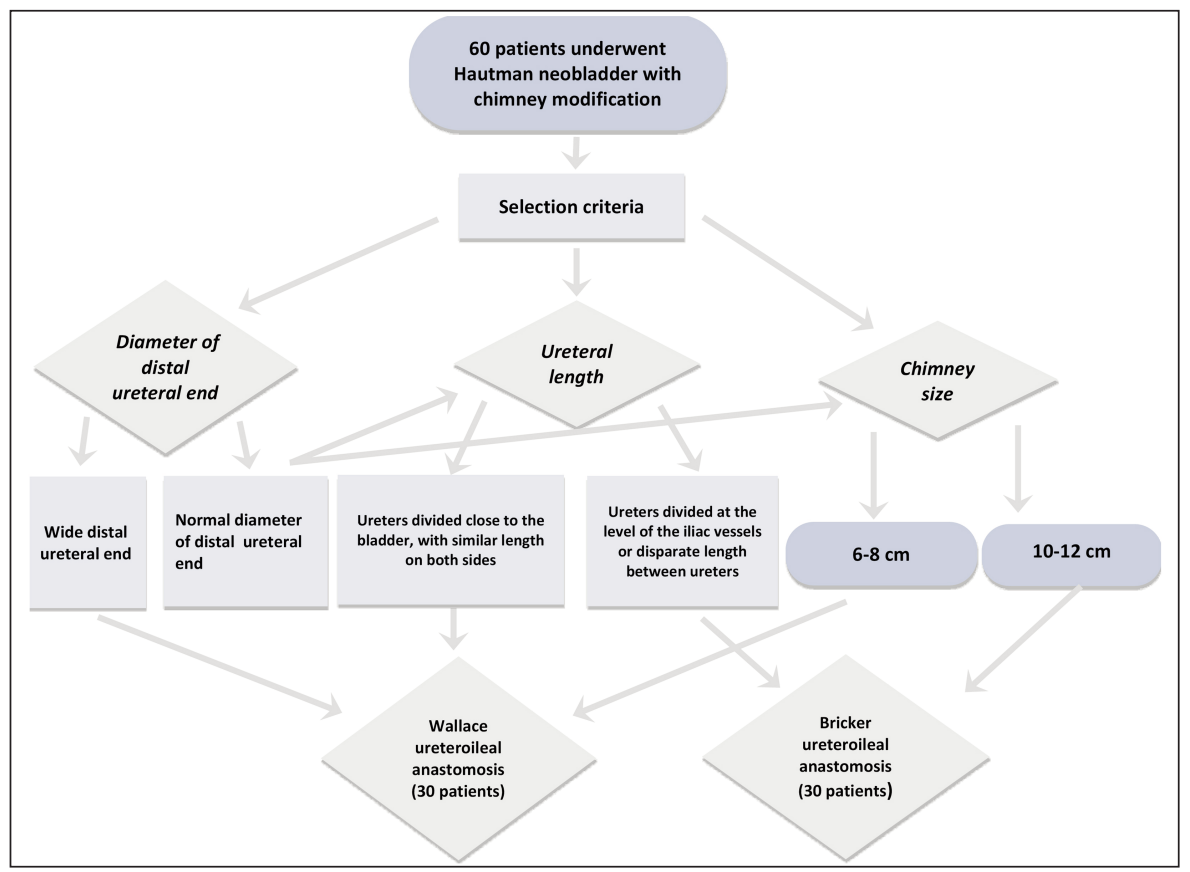

Clinical Centre of Serbia and conducted in accordance with the principles of the Declaration of Helsinki from the World Medical Association. The surgery comprised RC with standard pelvic lymph node dissection (PLND), which was followed by reconstruction of Hautmann neobladder with chimney modification. Eligible patients were aged $\geq$ $30 \mathrm{yr}$ and had BCa clinical stage T2-T3/NO/MO. Patients were excluded if they had previous pelvic radiation, clinical stage T4 or N1-N3/M1, positive frozen-section urethral biopsy, extensive prior abdominal surgery, serum creatinine level of $>2.0 \mathrm{ng} / \mathrm{mL}$ and any history of upper urinary tract malignancy (4). Complications were reported according to the modified Clavien-Dindo classification system (18). Reservoir-related complications included obstructive or non-obstructive hydronephrosis, UIS, pyelonephritis, anastomotic leakage, metabolic acidosis and vesicoureteral reflux (VUR). UIS was diagnosed when there was evidence of obstruction on imaging (symptomatic hydronephrosis), worsening renal function or infection (18). Non-obstructive hydronephrosis was defined as a distended intrarenal collecting system on imaging without evidence of UIS or other mechanical obstruction and was confirmed by intravenous urography (IVU) or computed tomography (CT).

Of note, pyelonephritis was designated as a positive urine culture in association with foul smelling urine and fever (19). Perioperative outcomes were systematically and prospectively collected at surgery and during hospitalization and each complication classified as early $(<3$ months) or late ( $>3$ months after surgery). Patient interviews were conducted by medical doctors and according to European Association of Urology (EAU) guidelines on reporting and grading of complications (20).

In this study we aimed to establish an optimal technique selection strategy for ureteroenteric anastomosis (Bricker vs. Wallace) based on patient characteristics and evaluated according to difference in early and late perioperative complications and postoperative healthrelated quality of life (HRQOL).

\section{Outcomes measures and follow up}

Postoperatively, all patients were placed on the identical treatment pathway and follow-ups were scheduled every 3-4 months during the first year and semi-annually in the second (19). Renal function was measured by serum creatinine, hydronephrosis was examined by abdominal ultrasound or computed tomography, and VUR was assessed by voiding cystography (6). Diagnostic imaging (kidney ultrasound, CT abdomen/pelvis and chest radiography) was performed annually or when clinically indicated. Abdominal ultrasound (US) was performed immediately before discharge of patients to deter- 
Table 1.

Clinicopathological features and perioperative outcomes between group I and II.

\begin{tabular}{|c|c|c|c|}
\hline \multicolumn{4}{|c|}{ Mean (SD)/Percentage (\%) } \\
\hline Clinicopathological characteristics & Group I $(n=30)$ & Group II $(n=30)$ & P value \\
\hline Age (years) & $63(7.2)$ & $68(6.6)$ & 0.6 \\
\hline BMI, $\mathrm{kg} / \mathrm{m}^{2}$, mean (SD) & $27.2(2.6)$ & $26.1(3.2)$ & 0.8 \\
\hline Male, n (\%) & $22(73.3)$ & $24(80.0)$ & 0.2 \\
\hline Female, $n(\%)$ & $8(26.6)$ & $6(20)$ & 0.1 \\
\hline \multicolumn{4}{|l|}{ ASA score, $n(\%)$} \\
\hline 2 & $17(56.6)$ & $18(62)$ & 0.3 \\
\hline$\geq 3$ & $13(43.3)$ & $11(38)$ & 0.09 \\
\hline \multicolumn{4}{|l|}{ Pathologic stage, n (\%) } \\
\hline $\mathrm{T} 2$ & $23(76.6)$ & $25(83.3)$ & 0.1 \\
\hline ТЗ & $7(23.4)^{*}$ & $4(13.3)$ & 0.03 \\
\hline LNP patients, $n(\%)$ & $4(13.3)$ & $5(16.6)$ & 0.7 \\
\hline Operative time (min), SD & $270(42.3)$ & $240(33.6)$ & 0.3 \\
\hline Estimated blood loss (ml), SD & $340(150)$ & $400(210)$ & 0.06 \\
\hline Hospital stay (days), SD & $18(4.6)$ & $19(3.4)$ & 0.6 \\
\hline Transfusion rate, $n(\%)$ & $7(23.3)$ & $5(16.6)$ & 0.08 \\
\hline
\end{tabular}

mine the pouch capacity and post voiding residue (PVR). The acidosis was monitored using the base excess by venous blood gas analysis, initially every three days followed by weekly, depending on the blood gas values.

The European Organization for the Research and Treatment of Cancer (EORTC) Quality-of-Life Core Questionnaire (QLQ-C30) version 3 was used to measure HRQOL $(21,22)$.

Continence rates and time intervals between clear intermittent catheterizations (CICs) obtained at the end of 2-year follow up were recorded. CIC was recommended for patients with a postvoid residual volume (PVR) of $>150 \mathrm{~mL}$

\section{Statistical analysis}

Statistical analysis was performed with SPPS v16.0 (SPPS, Chicago, IL, USA). Blood loss, operative time, and time to discharge (hospital stay) were assessed as continuous variables and tested for normality using the Kolmogorov test. The Student $\mathrm{T}$ test and Mann Whitney U test were used to determine statistical significance. The difference between obtained values was considered significant when $p<0.05$. Descriptive statistics such as mean (SD) values and percentages, generated with SPSS, were also included.

\section{RESULTS}

Clinicopathological features and perioperative outcomes are summarized in Table 1.

The two groups were similar for gender, age, ASA class and BMI. All patients had transitional cell carcinoma and the tumor stage ranged from $\mathrm{T} 2$ to T3 N0-3/M0. The followup time for the entire cohort was 2 years.

\section{Table 2.}

The mean operative time was $270 \pm 42.3 \mathrm{~min}$ and $240 \pm$ $33.6 \mathrm{~min}$ in the first and second group respectively $(\mathrm{p}=$ 0.3). The distribution of postoperative complications is shown in table 2. A total of 135 complications were recorded in 40/60 (66.6\%) patients. 105 complications (77.7\%) occurred in the first 90 days, with the remaining 30 complications (22.2\%) occurring between 90 days and one year postoperatively. The majority of complications $(44 / 60,73.3 \%)$ were classified as low-grade with $41.6 \%$ in Grade I and 31.6\% in Grade II. High-grade (ClavienDindo Grade III-V) complications were seen in 10/60 (16.6\%) patients. Grade III, IV and V complications were observed in $11.6 \%, 1.6 \%$ and $3.3 \%$ of the patients, respectively (Table 3). The overall mortality rate was 3.3\% $(2 / 60)$. High-grade complications were less in Wallace group as compared to Bricker group, and the difference was significant $(3 / 30,10 \%$ vs. $6 / 30,20 \%, p=0.03)$.

Following 3 months, hydronephrosis was observed in eight patients (26.6\%) in group I and six (20\%) in group II, $(\mathrm{p}=0.2)$ (grade I-III Clavien). Consequent to hydronephrosis, UIS was seen in two ureters $(6.6 \%)$ in group I but none in group II (grade III Clavien). Moreover, one out of two patients with UIS required surgical treatment (grade IIIb Clavien). These differences were statistically significant $(\mathrm{p}=0.0063)$.

Additionally, the anastomotic leakage rate was higher in the first group, although not significantly $(6.6 \%$ vs. $3.3 \%$, $\mathrm{p}=0.06$ ) (grade I/IIIa Clavien).

Postoperative complications of 60 patients with muscle invasive bladder cancer who underwent radical cystectomy and modified Hautmann neobladder with Bricker (group I) or Wallace (group II) ureteroileal anastomosis.

\begin{tabular}{|c|c|c|c|c|c|}
\hline \multicolumn{6}{|c|}{ Mean (SD)/Percentage (\%) } \\
\hline \multirow[t]{2}{*}{ Clinicopathological characteristics } & \multicolumn{2}{|c|}{ Group I ( $=30)$} & \multicolumn{2}{|c|}{ Group II ( $=30$ ) } & \multirow[t]{2}{*}{$P$ value } \\
\hline & Early & Late & Early & Late & \\
\hline Paralitic ileus, $n(\%)$ & $8(26.6)$ & 0 & $9(30)$ & 0 & 0.7 \\
\hline Wound infections, n (\%) & $2(6.6)$ & $1(3.3)$ & $1(3.3)$ & $3(10)$ & 0.4 \\
\hline Blood transfusions for anemia, n (\%) & $9(30)$ & 0 & $10(33.3)$ & 0 & 0.5 \\
\hline Pelvic hematoma, n (\%) & $2(6.6)$ & 0 & $1(3.3)$ & 0 & 0.1 \\
\hline Lymphorrhea, n (\%) & $6(20)$ & $1(3.3)$ & $4(13.3)$ & $2(6.6)$ & 0.08 \\
\hline Pneumonia, n (\%) & 0 & $4(13.3)$ & $1(3.3)$ & $3(10)$ & 0.1 \\
\hline Reservoir related complications & Early & Late & Early & Late & \\
\hline Renal insufficiency, n (\%) & 0 & $1(3.3)$ & 0 & 0 & 0.07 \\
\hline Vesicoureteral reflux (VUR), n (\%) & $4(13.3)$ & $3(10)^{*}$ & $3(10)$ & $1(3.3)$ & 0.03 \\
\hline - Grade I & 2 & 2 & 2 & 1 & \\
\hline - Grade II & 1 & 1 & 1 & 0 & \\
\hline - Grade III & 1 & 0 & 0 & 0 & \\
\hline - Grade IV & 0 & 0 & 0 & 0 & \\
\hline Hydronephrosis, n (\%) & $8(26.6)$ & $1(3.3)$ & $6(20)$ & $3(10)^{*}$ & 0.02 \\
\hline - Unilateral & 8 & 1 & 0 & 2 & \\
\hline - Bilateral & 0 & 0 & 6 & 2 & \\
\hline Pyelonephritis, $n(\%)$ & $4(13.3)$ & 0 & $5(16.6)$ & 0 & 0.4 \\
\hline - i.va antibiotics only & 1 & 0 & 2 & 0 & \\
\hline - Oral antibiotics only & 3 & 0 & 3 & 0 & \\
\hline Anastomotic leakage rate, n (\%) & $2(6.6)$ & 0 & $1(3.3)$ & 0 & 0.09 \\
\hline Anastomotic stricture rate (UIS), n (\%) & $2(6.6)^{*}$ & $1(3.3)$ & 0 & 0 & 0.04 \\
\hline Metabolic acidosis, n (\%) & $6(20)$ & $1(3.3)$ & $5(16.6)$ & $2(6.6)^{*}$ & 0.04 \\
\hline
\end{tabular}


Table 3.

Classification of postoperative complications and treatment options for reservoir-related complications after radical cystectomy and construction of modified Hautmann reservoir with Bricker (group I) or Wallace (group II) ureteroileal anastomosis.

\begin{tabular}{|c|c|c|c|c|c|}
\hline \multirow{2}{*}{$\begin{array}{l}\text { Postoperative complications } \\
\text { \& treatment }\end{array}$} & \multicolumn{2}{|c|}{ Group I ( $n=30$ ) } & \multicolumn{2}{|c|}{ Group II ( $n=30$ ) } & \multirow[t]{2}{*}{$P$ value } \\
\hline & Early & Late & Early & Late & \\
\hline Clavien-Dindo classification & $19(63.3)^{*}$ & $9(30)$ & $16(53.3)$ & $10(33.3)$ & 0.03 \\
\hline Grade I & $8(26.6)$ & $4(13.3)$ & $9(30)$ & $4(13.3)$ & \\
\hline Grade II & $7(23.3)$ & $2(6.6)$ & $5(16.6)$ & $5(16.6)^{*}$ & \\
\hline Grade III (IIIa/IIIb) & $4(13.3)^{*}$ & $1(3.3)$ & $2(6.6)$ & 0 & \\
\hline Grade IV (IVa/IVb) & 0 & $1(3.3)$ & 0 & 0 & \\
\hline Grade V (death) & 0 & $1(3.3)$ & 0 & $1(3.3)$ & \\
\hline Treatment, $n(\%)$ & $15(50)^{*}$ & $7(23.3)^{*}$ & $6(20)$ & $4(13.3)$ & 0.01 \\
\hline Antegrade stent placement & 1 & 0 & 0 & 0 & \\
\hline Percutaneus nephrostomy (PCN) & 1 & 1 & 1 & 0 & \\
\hline Balloon dilatation of strictures & 2 & 0 & 0 & 0 & \\
\hline Surgical repair of strictures & 1 & 0 & 0 & 0 & \\
\hline Intraabdominal drainage & 2 & 0 & 0 & 0 & \\
\hline Intermitent catheterization (CICS) & 8 & 6 & 5 & 4 & \\
\hline
\end{tabular}

Nine patients (15\%) required interventions under general or local anesthesia for the management of high-grade ( $\geq$ III) complications. Four patients from the first group required invasive treatment of early reservoir-related complications (13.3\%), which was significantly higher compared to the Wallace group $(3.3 \% ; \mathrm{p}=0.01)$. Three patients underwent percutaneous nephrostomy for ureteroileal anastomotic stricture or anastomotic leak in both groups, whereas two patients from the first group had percutaneous drainage for lymphocele or pelvic collection. Only one patient from the Bricker group developed local tumor recurrence (3.3\%) (grade IIIa), which was treated endoscopically (Table 3). Neoadjuvant chemotherapy was performed in 16 patients (27.1\%), while adjuvant treatment received only one examinee (1.7\%).

At the end of the 2-year follow-up, two patients with VUR in group I had improved after CICs (grade I Clavien). In addition, five out of six patients with hydronephrosis had improved with or without treatment. However, one case with UIS - related unilateral hydronephrosis resulted in a non-functional kidney despite the treatment for the stricture (grade IV Clavien). Serum creatinine was less than $1.4 \mathrm{mg} / \mathrm{dl}$ preoperatively in all patients and it remained within the normal ranges, during the follow-up in both groups, except in the patient with kidney failure.

Complete daytime continence at one year was achieved in 28 patients (93.3\%) in the first group and 26 patients $(89.6 \%)$ in the second group, with no statistical differences however $(p>0.05)$. Complete night time continence was achieved in 24 (80\%) and 25 patients (86.2\%), respectively $(p>0.05)$. Patient self-rated emotional and social functional scales were similar between groups.

\section{Discussion}

The first description of Hautmann neobladder with chimney modification was published by Lipper and Theodorescu (2) consisting of a 5-10 cm isoperistaltic chimney with an end-to-side ureteroileal anastomosis. The study included three patients with short follow-up; no postoperative complications were reported, demonstrating that the technique employed was a promising modification to the original Hautmann neobladder. In 2000 , a more comprehensive study was performed on 50 patients with invasive $\mathrm{BCa}$ (3), using 8-12 cm tubularised isoperistaltic ileal chimney. This technique proved to be safe and feasible, easy to perform and created a reliable ureterointestinal anastomosis (Bricker) without tension, which resulted in a relatively low UIS rate (6\%). On the other hand, Hautmann et al. (23) reported that freely refluxing Wallace anastomosis to the afferent limb of the orthotopic reservoir has the lowest non-tumor related anastomotic stricture rate (5.4\% compared to $16.3 \%$ using Bricker technique). Furthermore, Kouba et al. (12) revealed a statistically significant difference in UIS rate between Bricker and Wallace anastomotic techniques (3.7\% vs. 0 ), in favour with the latter. Despite these results, the success and complications of two techniques are still debatable and no definite conclusion regarding the optimal anastomotic technique for orthotopic diversion has been made. The reason for this may be the lack of clear selection criteria for each anastomotic technique, instead of simple surgeon preference $(12,24)$. A recent study (17), suggesting an individualized selection strategy for deciding upon the type of uretero-ileal anastomosis (Bricker vs. Wallace), showed acceptable low rate of ureteral strictures (3.1\%) and confirmed clinical reliability of research. The technique selection was based on several individual patient factors, including tumor characteristics, ureteral anomalies and ureteral length. In our study, however, chimney size and diameter of distal ureter, together with ureteral length after retro-sigmoidal tunneling were considered as selection criteria to decide upon the type of ureteroileal anastomosis. The results we reported here revealed higher incidence of UIS using Bricker technique (6.6\%), after three months follow-up; on the other hand, this complication was not detected using Wallace anastomosis on shortened tubularised isoperistaltic chimney. Since this type of stricture remains the most challenging and difficult of all ureteral strictures to treat $(25,26)$, any technical modification that aims to decrease or prevent UIS is recommended (17). Our modified Wallace technique consisted of longer ureteral spatulation and short Chimney, seemed to be effective in reducing the occurrence of both UIS and anastomotic leakage during followup period. Moreover, a shorter chimney may also play a role in reflux prevention, due to the fact that a shorter limb allows the use of longer segments of the lower ureters that participate in reflux prevention (27).

All these findings together bolster the assertion that proper patient selection and meticulous ureteral handling of distal ureter, as well as a shorter intestinal chimney with end-to-end running suture ureteroileal anastomosis, may be essential to minimize the risk of postoperative reservoir-related complications. 
Oncologic factors were an important consideration in our series. Although the Wallace technique has the lowest rate of UIS, it not recommended for patients with increased risk of recurrent tumors (bladder CIS) $(4,11)$.

In our study, however, patients with multifocal bladder CIS were selected exclusively for Bricker anastomotic technique, after obtaining negative frozen-section urethral biopsy. Therefore, oncologic limitations of direct end-to-end ureteroileal anastomosis were clinically insignificant in our cohort with conclusion that Wallace technique may become the preferred anastomotic approach, in properly selected patients.

It is questionable, however, why the Bricker group was associated with high postoperative complications rate (Clavien-Dindo grade III-13.3\%), where incidence of UIS was higher than expected $(1,3,23)$. Since the obesity may impair the outcome of ureteroileal anastomosis after RC (17), we assumed that higher BMI of patients within Bricker group, associated with short mesentery and extensive dissection of the distal left ureter, led to significantly higher rate of UIS, comparing to Wallace group. Incidence of CICs after neobladder construction is generally $4-25 \%$ in males and up to $53 \%$ in females $(28,29)$. In our study, however, $16.9 \%$ of patients still required CICs at the end of first year. Nevertheless, during the initial three months post-surgery, hydronephrosis had improved after CICs in 50\% of patients within the second group, whereas only one patient required an invasive procedure for the treatment of hydronephrosis (PCN).

The limitations of this study are the small size of groups of patients and the short follow-up periods. Despite that, we found an acceptable rate of ureteroenteric strictures, VUR and anastomotic leakage, lower than that found in the conventional technique. Furthermore, our research was conducted with no clear protocol for administration of neoadjuvant or adjuvant chemotherapy, as this was left to the discretion of the uro-oncologist board. In addition, the unusually high rate of anastomotic leakage in patients within the first group could lead to research bias regarding effectiveness of our modified Hautmann neobladder with Wallace anastomotic technique.

Single surgeon experience could be the major reason for this bias, which should be addressed by involving other highly trained surgeons.

\section{Conclusions}

Our preliminary outcomes demonstrated that this patient-based selection strategy for ureteroileal anastomosis in orthotopic urinary diversion after RC seems to be clinically reliable and favors Wallace anastomotic technique over the Bricker approach.

\section{References}

1. Hautmann RE, Abol-Enein H, Davidsson T, et al. ICUD-EAU International Consultation on Bladder Cancer 2012: urinary diversion. Eur Urol. 2013; 63:67-80.

2. Lippert MC, Theodorescu D. The Hautmann neobladder with a chimney: a versatile modification. J Urol. 1997; 158:1510-2.

3. Hollowell CM, Christiano AP, Steinberg GD. Technique of
Hautmann ileal neobladder with chimney modification: interim results in 50 patients. J Urol. 2000; 163:47-50.

4. Sevin G, Soyupek S, Armagan A, et al. Ileal orthotopic neobladder (modified Hautmann) via a shorter detubularised ileal segment: experience and results. BJU Int. 2004; 94:355-59.

5. Bianchi G, Sighinolfi MC, Pirola GM, Micali S. Studer orthotopic neobladder: a modified surgical technique. Urology. 2016; 88:22225.

6. Shigemura K, Yamanaka N, Imanishi O, Yamashita M. Wallace direct versus anti-reflux Le Duc ureteroileal anastomosis: comparative analysis in modified Studer orthotopic neobladder reconstructions. Int J Urol. 2012; 19:49-53.

7. Studer UE, Burkhard FC, Schumacher M, et al. Twenty years experience with an ileal orthotopic low-pressure bladder substitute: lessons to be learned. J Urol. 2006; 176:161-66.

8. Lypczinski W, Glazar B, Bak M, et al. Strategy in preventing of uretero-intestinal anastomosis strictures in patients with low-pressure intestinal neobladder. Przegl Lek. 2012; 69:181-83.

9. Helmy Aly A, Ezzat A, Hamed A. Orthotopic neobladder reconstruction after radical cystectomy in patients with a solitary functioning kidney: clinical outcome and evaluation. J Egypt Natl Canc Inst. 2011; 23:133-40.

10. Micali S, De Carli P, Milano R, et al. Double-J ureteral stents: an alternative to external urinary stents in orthotopic bladder substitution. Eur Urol. 2001; 39:575-79.

11. McDouglas WS. Use of intestinal segments and urinary diversion. In: Walsh PC, Retik AB, Vaughan ED Jr, Wein AJ (eds). Campbell's Urology. Saunders, Philadelphia, PA, 2002; pp. 3745-88.

12. Kouba E, Sands M, Lentz A, et al. A comparison of the Bricker versus Wallace ureteroileal anastomosis in patients undergoing urinary diversion for bladder cancer. J Urol. 2007; 178:945-48.

13. Pantuck AJ, Han KR, Perrotti M, et al. Uretroenteric anastomosis in continent urinary diversion: long-term results and complications of direct versus nonrefluxing techniques. J Urol. 2000; 163:450-55.

14. Hautmann RE, de Petriconi RC, Volkmer BG. 25 years of experience with 1000 neobladders: long-term complications. J Urol. 2011; 185:2207-12.

15. Hautmann RE. Surgery illustrated - surgical atlas ileal neobladder. BJU Int. 2010; 105:1024-35.

16. Djordjevic D, Vukovic M. Functional results of Hautmann neobladder with chimney modification and Wallace ureteroileal anastomosis: initial experience with 22 patients. Int Braz J Urol. 2021; 47:426-435.

17. Liu L, Chen M, Li Y, et al. Technique selection of bricker or wallace ureteroileal anastomosis in ileal conduit urinary diversion: a strategy based on patients characteristics. Ann Surg Oncol. 2014; 21:2808-12.

18. Kanno T, Inoue T, Kawakita M, et al. Perioperative and oncological outcomes of laparoscopic radical cystectomy with intracorporeal versus extracorporeal ileal conduit: A matched-pair comparison in a multicenter cohort in Japan. Int J Urol 2020; 27:559-565.

19. Al Hussein Al Awamlh B, Wang LC, et al. Is continent cutaneous urinary diversion a suitable alternative to orthotopic bladder substitute and ileal conduit after cystectomy. BJU Int. 2015; 116:805-14.

20. Mitropoulos D, Artibani W, Graefen M, et al. Reporting and grading of complications after urologic surgical procedures: an ad hoc EAU guidelines panel assessment and recommendations. Eur Urol. 2012; 61:341-9. 
21. Aaronson NK, Ahmedzai S, Bergman B, et al. The European Organization for Research and Treatment of Cancer QLQ-C30: a quality-of-life instrument for use in international clinical trials in oncology. J Natl Cancer Inst. 1993; 85:365-376.

22. Singh V, Yadav R, Sinha RJ, Gupta DK. Prospective comparison of quality of life outcomes between ileal conduit urinary diversion and orthotopic neobladder reconstruction after radical cystectomy: a statistical model. BJU Int. 2014; 113:726-732.

23. Hautmann RE, Volkmer BG, Schumacher MC, et al. Long-term results of standard procedures in urology: the ileal neobladder. World J Urol. 2006; 24:305-314.

24. Evangelidis A, Lee EK, Karellas ME, et al. Evaluation of ureterointestinal anastomosis: Wallace vs. Bricker. J Urol. 2006; 175:1755-8.
25. Kurzer E, Leveillee RJ. Endoscopic management of ureterointestinal strictures after radical cystectomy. J Endourol. 2005; 19:677-82.

26. Farnham SB, Cookson MS. Surgical complications of urinary diversion. World J Urol. 2004; 22:157-67.

27. Hassan Abol-Enein, Nuzhat Faruqui, Nashwa Barakat, Shokeir AA. Does the afferent tubular segment in an orthotopic bladder substitution compromise ureteric antireflux properties? An experimental study in dogs. Arab J Urol. 2012; 10:125-30.

28. Hautmann RE, Paiss T, de Petriconi R. The ileal neobladder in women: 9 years of experience with 18 patients. J Urol. 1996;155:76-81.

29. Ali-el-Dein B, el-Sobky E, Hohenfellner M, Ghoneim MA. Orthotopic bladder substitution in women: functional evaluation. J Urol. 1999; 161:1875-80.

\section{Correspondence}

Dejan Djordjevic, MD, PhD, Urologist

dejanurl@gmail.com

Svetomir Dragicevic, MD, Urologist

dejanur@@mail.com

Urology Clinic, Euromedic General Hospital

Bulevar Umetnosti 29, 11000 Belgrade (Serbia)

Marko Vukovic, MD (Corresponding Author)

marko.vukovic09@gmail.com

Department of Urology, Clinical centre of Montenegro

Ljubljanska bb, 81000 Podgorica (Montenegro) 